\title{
Reducing risks from coronavirus transmission in the home-the role of viral load
}

Paul Little and colleagues call for better promotion of simple measures that can help reduce the spread and severity of infection among those living with people who have covid-19

\author{
Paul Little professor ${ }^{1}$, Robert C Read professor ${ }^{2}$, Richard Amlôt scientific programme ${ }^{3}$ leader ${ }^{3}$, Tim \\ Chadborn head of group ${ }^{4}$, Cathy Rice public contributor ${ }^{5}$, Jennifer Bostock research adviser ${ }^{6}$, Lucy \\ Yardley professor $^{78}$
}

${ }^{1}$ Primary Care Population Sciences and Medical Education Unit, Faculty of Medicine, University of Southampton, Southampton, UK; ${ }^{2} \mathrm{NIHR}$ Southampton Biomedical Research Centre, University Hospital Southampton, Faculty of Medicine and Institute for Life Sciences, University of Southampton, Southampton, UK; ${ }^{3}$ Behavioural Science Team, Emergency Response Department, Public Health England, London, UK ; ${ }^{4}$ Behavioural Insights, Public Health England, London, UK ; ${ }^{5}$ Bristol, UK ; ${ }^{6}$ Nuffield Department of Population Health, University of Oxford, Oxford UK; ${ }^{7}$ Department of Psychology, University of Southampton, Southampton, UK; ${ }^{8}$ School of Psychological Science, University of Bristol, Bristol, UK

Most people with covid-19 are cared for at home, increasing the likely exposure of household members. Although the evidence is limited, high infection rates among health workers have been attributed to more frequent contact with infected patients, and higher viral load — the size of the infecting dose of virus. This has led to demands for better personal protection equipment (PPE). Less attention, however, has been given to family members and others caring for people with covid-19 in the community. Providing them with the same level of PPE as in hospitals is not practicable, but promotion of simple evidence based interventions may lower the risk of infection transmission and help reduce morbidity and demand on hospitals.

\section{Transmission in home and community}

The long incubation and high presymptomatic infectivity of covid-19 makes transmission between family members a particular risk. Modelling of viral shedding in 94 patients with covid-19 and 77 transmission pairs suggests that the highest viral load is at or just before symptom onset, with $44 \%$ of transmission occurring before symptoms. ${ }^{1}$

Public health advice recommends isolation of symptomatic household members, but this can be difficult, particularly in small flats with shared facilities. Motivation to overcome these difficulties may not be high enough if members of the public are sceptical about reducing transmission in the home and unaware that the illness of other family members may be more severe if they do not reduce their level of exposure.

The medical community is commendably reluctant to make recommendations in the absence of evidence. An expert team that reviewed the evidence for viral load concluded that until the evidence is more conclusive: "As our grandfathers used to say, when you do not know what is going on, do nothing." However, given that measures to reduce the viral load from exposure to symptomatic household members have little risk of harm, the precautionary principle suggests that we should be promoting them. It is difficult to get good dose-response data, but it seems prudent not to treat absence of direct evidence in the pandemic as evidence of absence, particularly given evidence from other viral infections from both animal and human models, and trial evidence for reducing the transmission among families in non-pandemic years. Greater awareness of the potential risk from viral load may help motivate family members to pay sufficient attention to protecting themselves despite their concern for sick family members.

\section{Evidence for viral load effect}

It is intuitive that viral load should influence the incidence and severity of disease. The main problem is that measuring the viable infecting dose of the virus in people is extremely challenging: contemporary measures of viral density, viability, and viral contamination are all hard to obtain. Establishing the relation between infecting dose and the likelihood of developing disease is therefore difficult.

The challenge of establishing the infecting dose is complicated by environmental contamination. Experience with Middle East respiratory syndrome (MERS), caused by another coronavirus (MERS-CoV), suggests that environmental contamination with SARS-CoV-2 is likely to be high, ${ }^{3}$ and this is supported by recent case reports of extensive environmental contamination from patients with covid-19. Indirect evidence, including from 
animal models and epidemiological studies also provides support.

\section{Animal models}

Although the infecting dose from a combination of droplets and environmental contamination cannot be easily measured, high quality experiments under controlled conditions in animal models can provide indirect evidence. We are not aware of infecting dose experiments with animal models of covid-19, but animal models of other viral infections show that variation in the infecting dose determines how many animals get infected and how severe the illness is.

A model of African swine flu virus shows a clear dose-response relation between the infecting dose and disease in the animal. ${ }^{56}$ Likewise, a strong dose-response effect is found in the animal model for haemopoietic necrosis virus. ${ }^{6}$ A dose-response has also been shown in mouse models with several strains of SARS-CoV-1, which is closely related the virus causing covid-19 (SARS-CoV-2). The infectivity varies between different strains of the virus, which modifies the shape of the dose-response curve, but nevertheless consistent dose-response relations are observed with the severity of the infection. ${ }^{78}$

\section{Defective viral genomes}

One of the key factors in determining how severe an infection becomes is also the extent to which defective viral genomes are produced. These effectively reduce the infecting doses during the early part of an infection by competing with non-defective genomes. ${ }^{9}$ The greater the abundance of viruses with defective genomes within an infecting inoculum, the better the clinical outcome $^{10}$ : genomic analysis of viruses isolated from previously healthy people requiring admission to the intensive care unit with influenza A infection, those not requiring intensive care, and those who died (who also had underlying medical conditions) showed that defective genomes were associated with fewer severe or fatal outcomes.

\section{Epidemiology of serious viral infections}

The evidence from other similar serious viral infections also suggests the infecting viral load may be important. A retrospective study of survivors of Ebola from the Kerry Town treatment centre in Sierra Leone, investigated disease in more than 933 family members (those who had died, those surviving, and those not infected). The severity of infecting dose was graded according to the history of exposure. Although there was no clear relation with mortality, perhaps because of the mediating effect of treatment at the treatment centre, the study found a very strong linear relation with the likelihood of infection developing, ranging from $80 \%$ likelihood of getting the disease with the highest infecting dose (direct contact with body of someone who had died) and $10 \%$ with the lowest dose (no contact). ${ }^{11}$

During the 2003 SARS outbreak older age, comorbidities (adjusted hazard ratio (HR) 3.36, 95\% confidence interval 1.44 to 7.82), and higher initial viral levels in nasopharyngeal specimens (adjusted HR 1.21 per $\log _{10}$ increase in number of RNA copies/mL, 95\% CI 1.06 to 1.39 ) were associated with worse survival. ${ }^{12}$ Viral level 10 days after the onset of symptoms was associated with a series of poor clinical markers (oxygen desaturation, mechanical ventilation) and death. ${ }^{13}$ Recent data from covid-19 have shown that those with severe infections had viral levels 60 times higher at presentation than those with mild disease. ${ }^{14}$ Although the levels of virus once the disease has started will be in part a function of the immune response of the patient, the size of the initial viral load is likely to be a contributing factor, allowing immune defences to be more easily over-run.

The difference in case fatality rates in the three waves of the Spanish flu pandemic of 1918-19 can be explained by the number of simultaneous contacts a susceptible person had with infected people (the more contact the higher the infectious doses). ${ }^{15}$ However, in a detailed study modelling influenza virus transmission within households in 2008-12, infectivity was proportional to viral load but viral load alone provided a poor fit to the models. ${ }^{16}$ Clearly we need to better understand the relation between infecting dose and other prognostic factors in modifying the immune response and clinical outcome (age, comorbidity, etc).

\section{Pragmatic evidence that could help carers}

We are aware of only one behavioural intervention that is proved to reduce virus transmission within households and is suitable for rapid dissemination in a pandemic. Two of us (PL and LY) were involved in a randomised trial of Germ Defence, a website that provides advice on infection control measures and helps users think about when and how to carry out key infection control behaviours such as handwashing and cleaning, avoiding sharing rooms and surfaces, managing incoming deliveries, and ventilating rooms. ${ }^{17}$ This could supplement public health advice on infection control in the home since it uses behaviour change techniques to help people implement this advice (box 1).

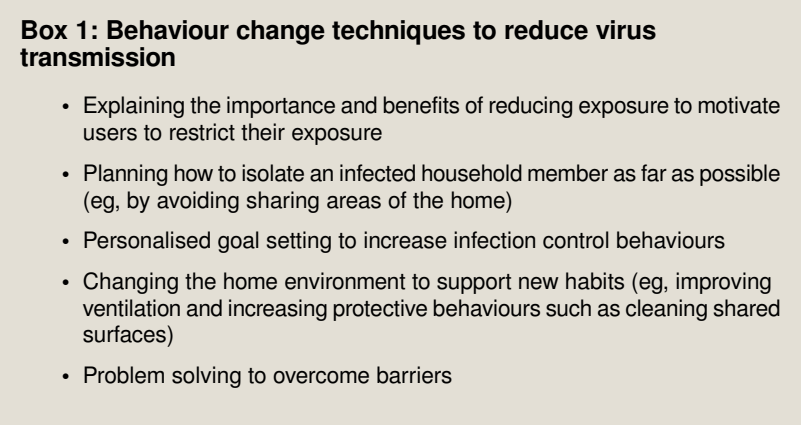

Germ Defence was trialled in 20066 people during the H1N1 pandemic and subsequent seasonal flu years ${ }^{17}$ and reduced the number of respiratory infections (mean number of infections $0.84 v 1.09$ in the control group, hazard ratio $0.75,95 \%$ confidence intervals 0.72 to 0.79 ). Infection transmission among family members was lower in the intervention group (hazard ratio $0.79,95 \%$ confidence interval 0.74 to 0.83 ), and there was a modest reduction in severity of infections (mean number of days of moderately bad illness 3.9 (median 2 days) in intervention versus 4.5 (3) days in the control group). Reductions were also observed in gastrointestinal infections, GP consultations, and antibiotic prescriptions.

The team has been funded by UK Research and Innovation to adapt this intervention for covid-19 and disseminate it nationally and internationally. It has already been translated into over 20 languages for this purpose (see www.germdefence.org/). Germ Defence may help limit transmission of covid-19 as well as the other viruses that are still causing the majority of respiratory illnesses in the current pandemic, even in secondary care settings. ${ }^{18}$ Other viruses may also be important given recent evidence that coinfection with other viruses occurs in more than $20 \%$ of cases. $^{19}$ 


\section{Conclusion}

Care is needed when extrapolating evidence from other disease, but viral load is likely to be important for covid-19. The precautionary principle suggests that people caring for household members who are unwell should be encouraged to take measures to reduce infecting viral load in order to reduce the incidence and severity of infection. Promoting infection control measures in the community is a priority for the UK government and will continue to be so as "stay at home" policies are lifted.

Dissemination of evidence based behavioural interventions may help increase adoption of public health advice and reduce viral load.

\section{Key messages}

Government policy is aimed at reducing transmission of covid- 19 between family units, but less attention has been given to transmission between family members

Evidence from controlled experiments in animal models, viral genome studies, and other epidemics suggests the infecting viral load may be important

A web based intervention has been shown to reduce incidence, transmission, and severity of seasonal flu

Use of such behavioural interventions could support public health advice to improve infection control in families

Contributors and sources: PL is a clinical GP academic with expertise in infections in primary care and the community. LY is a behavioural scientist with expertise in digital support for illness management. RCR is an academic infectious disease physician. TC has a split role as head of behavioural insights and evaluation lead at Public Health England and expert analyst in the Systems Unit at the Cabinet Office. Since surviving a stroke, $\mathrm{CR}$ has undertaken public involvement in a wide range of health research. JB is a public adviser to the covid-19 Germ Defence project, a member of the Healthcare Infection Society and led the public involvement of the evaluation of the implementation of the UK's antimicrobial resistance strategy. RA is codirector of the NIHR Health Protection Research Unit in Behavioural Science and Evaluation of Interventions and a visiting professor of practice in the psychology of health protection at King's College London. All authors were involved in discussing, writing, and revising the article. $\mathrm{PL}$ is the guarantor.

Public and patient involvement: CR and JB participated in discussing, writing, and revising this article.

Competing interests: We have read and understood BMJ policy on declaration of interests and declare that PL was principal investigator and LY participated in the trial of Germ Defence funded by the Medical Research Council. All other authors except RCR are part of the team that is expanding and disseminating Germ Defence for covid-19, which LY is leading.

Provenance and peer review: Not commissioned; externally peer reviewed.

1 He X, Lau EHY, Wu P, et al. Temporal dynamics in viral shedding and transmissibility of COVID-19. Nat Med 2020. 10.1038/s41591-020-0869-5 32296168

2 Centre for Evidence Based Medicine. SARS-CoV-2 viral load and the severity of Covid-19, 26 Mar 2020. https://www.cebm.net/covid-19/sars-cov-2-viral-load-and-the-severity-ofcovid-19/

3 Bin SY, Heo JY, Song MS, etal . Environmental contamination and viral shedding in MERS patients during MERS-CoV outbreak in South Korea. Clin Infect Dis 2016;62:755-60. 10.1093/cid/civ1020 26679623

4 Ong SWX, Tan YK, Chia PY, et al. Air, surface environmental, and personal protective equipment contamination by severe acute respiratory syndrome coronavirus 2 (SARS-CoV-2) from a symptomatic patient. JAMA 2020. 10.1001/jama.2020.3227 32129805

5 Niederwerder MC, Stoian AMM, Rowland RRR, etal . Infectious dose of african swine fever virus when consumed naturally in liquid or feed. Emerg Infect Dis 2019;25:891-7. 10.3201/eid2505.181495 30761988

6 McKenney DG, Kurath G, Wargo AR. Characterization of infectious dose and lethal dose of two strains of infectious hematopoietic necrosis virus (IHNV). Virus Res 2016;214:80-9. 10.1016/j.virusres.2015.12.020 26752429

7 Roberts A, Lamirande EW, Vogel L, etal . Animal models and vaccines for SARS-CoV infection. Virus Res 2008;133:20-32. 10.1016/j.virusres.2007.03.025 17499378

8 Roberts A, Deming D, Paddock CD, etal . A mouse-adapted SARS-coronavirus causes disease and mortality in BALB/c mice. PLoS Pathog 2007;3:e5. 10.1371/journal.ppat.0030005 17222058

9 Vignuzzi M, López CB. Defective viral genomes are key drivers of the virus-host interaction. Nat Microbiol 2019;4:1075-87. 10.1038/s41564-019-0465-y 31160826

10 Vasilijevic J, Zamarreño N, Oliveros JC, etal . Reduced accumulation of defective viral genomes contributes to severe outcome in influenza virus infected patients. PLOS Pathog 2017;13:e1006650. 10.1371/journal.ppat.1006650 29023600

11 Bower $\mathrm{H}$, Smout E, Bangura MS, etal . Deaths, late deaths, and role of infecting dose in Ebola virus disease in Sierra Leone: retrospective cohort study. BMJ 2016;353:i2403. 10.1136/bmj.i2403 27188404

12 Chu CM, Poon LL, Cheng VC, etal . Initial viral load and the outcomes of SARS. CMAJ 2004;171:1349-52. 10.1503/cmaj.1040398 15557587

13 Hung IF, Cheng VC, Wu AK, etal. Viral loads in clinical specimens and SARS manifestations. Emerg Infect Dis 2004;10:1550-7. 10.3201/eid1009.040058 15498155

$14 \mathrm{Xu} \mathrm{T}$, Chen $\mathrm{C}$, Zhu Z, etal . Clinical features and dynamics of viral load in imported and non-imported patients with COVID-19. Int $J$ Infect Dis 2020;94:68-71; Epub ahead of print. 10.1016/.ijiid.2020.03.022 32179140

15 Paulo AC, Correia-Neves M, Domingos T, Murta AG, Pedrosa J. Influenza infectious dose may explain the high mortality of the second and third wave of 1918-1919 influenza pandemic. PLoS One 2010;5:e11655. 10.1371/journal.pone.0011655 20668679

16 Tsang TK, Cowling BJ, Fang VJ, etal . Influenza a virus shedding and infectivity in households. J Infect Dis 2015;212:1420-8. 10.1093/infdis/jiv225 25883385

17 Little P, Stuart B, Hobbs FD, etal . An internet-delivered handwashing intervention to modify influenza-like illness and respiratory infection transmission (PRIMIT): a primary care randomised trial. Lancet 2015;386:1631-9. 10.1016/S0140-6736(15)60127-1 26256072

18 Liu R, Han H, Liu F, etal . Positive rate of RT-PCR detection of SARS-CoV-2 infection in 4880 cases from one hospital in Wuhan, China, from Jan to Feb 2020. Clin Chim Acta 2020;505:172-5. 10.1016/j.cca.2020.03.009 32156607

19 Kim D, Quinn J, Pinsky B, Shah NH, Brown I. Rates of co-infection between SARS-CoV-2 and other respiratory pathogens. JAMA 2020. 10.1001/jama.2020.6266 32293646

Published by the BMJ Publishing Group Limited. For permission to use (where not already granted under a licence) please go to http://group.bmj.com/group/rights-licensing/ permissions 